Pacific

Journal of

Mathematics

SCOTT AND SWARUP'S REGULAR NEIGHBORHOOD AS A TREE OF CYLINDERS

VINCENT GUIRARDEL AND GILBERT LEVITT 


\title{
SCOTT AND SWARUP'S REGULAR NEIGHBORHOOD AS A TREE OF CYLINDERS
}

\author{
VINCENT GUIRARDEL AND GILBERT LEVITT
}

\begin{abstract}
Let $G$ be a finitely presented group. Scott and Swarup have constructed a canonical splitting of $G$ that encloses all almost invariant sets over virtually polycyclic subgroups of a given length. We give an alternative construction of this regular neighborhood by showing that it is the tree of cylinders of a JSJ splitting.
\end{abstract}

\section{Introduction}

Scott and Swarup [2003] have constructed a canonical graph of groups decomposition (or splitting) of a finitely presented group $G$; this splitting encloses all almost invariant sets over virtually polycyclic subgroups of a given length $n$ (the $\mathrm{VPC}_{n}$ groups), and in particular over virtually cyclic subgroups for $n=1$.

Almost invariant sets generalize splittings: Whereas a splitting is analogous to an embedded codimension-one submanifold of a manifold $M$, an almost invariant set is analogous to an immersed codimension-one submanifold.

Two splittings are compatible if they have a common refinement, in that both can be obtained from the refinement by collapsing some edges. For example, two splittings induced by disjoint embedded codimension-one submanifolds are compatible.

Enclosing is a generalization of this notion to almost invariant sets: Take, in the analogy above, two codimension-one submanifolds $F_{1}$ and $F_{2}$ of $M$ with $F_{1}$ immersed and $F_{2}$ embedded. Then $F_{1}$ is enclosed in a connected component of $M \backslash F_{2}$ if one can isotope $F_{1}$ into this component.

Scott and Swarup's construction is called the regular neighborhood of all almost invariant sets over $\mathrm{VPC}_{n}$ subgroups. This is analogous to the topological regular neighborhood of a finite union of (nondisjoint) immersed codimension-one submanifolds: It defines a splitting that encloses the initial submanifolds.

One main virtue of their splitting is that it is canonical: It is invariant under automorphisms of $G$. Because of this, it is often quite different from usual JSJ

MSC2000: primary 20E08; secondary 20F65, $20 \mathrm{E} 06$.

Keywords: JSJ decomposition, tree of cylinder, almost invariant set, torus theorem, canonical splitting, tree. 
splittings, which are unique only up to deformation. There the canonical object is the JSJ deformation space [Forester 2003; Guirardel and Levitt 2009].

The main reason for this rigidity is that the regular neighborhood is defined in terms of enclosing. Enclosing, like compatibility of splittings, is more rigid than domination, which is the basis for usual JSJ theory. For instance, any two splittings in Culler-Vogtmann's outer space dominate each other, but they are compatible if and only if they lie in a common simplex.

We have shown a general construction producing a canonical splitting $T_{c}$ from a canonical deformation space: the tree of cylinders [Guirardel and Levitt 2008]. It also enjoys strong compatibility properties. In this paper, we show that the splitting constructed by Scott and Swarup is a subdivision of the tree of cylinders of the usual JSJ deformation space.

More precisely, let $T_{J}$ be the Bass-Serre tree of a JSJ splitting of $G$ over $\mathrm{VPC}_{n}$ groups, as constructed for instance in [Dunwoody and Sageev 1999]. To construct the tree of cylinders, say that two edges are in the same cylinder if their stabilizers are commensurable. Cylinders are subtrees, and the tree $T_{c}$ dual to the covering of $T_{J}$ by cylinders is the tree of cylinders of $T_{J}$; see [Guirardel and Levitt 2008] or Section $2 b$ below.

Theorem 4.1. Let $G$ be a finitely presented group, and let $n \geq 1$. Assume that $G$ does not split over a $\mathrm{VPC}_{n-1}$ subgroup, and that $G$ is not $\mathrm{VPC}_{n+1}$. Let $T_{J}$ be a JSJ tree of $G$ over $\mathrm{VPC}_{n}$ subgroups, and let $T_{c}$ be its tree of cylinders for the commensurability relation.

Then the Bass-Serre tree of Scott and Swarup's regular neighborhood of all almost invariant subsets over $\mathrm{VPC}_{n}$ subgroups is equivariantly isomorphic to a subdivision of $T_{c}$.

This gives a new proof that this regular neighborhood is a tree. Deriving the regular neighborhood from a JSJ splitting, instead of building it from an abstract betweenness relation, seems to greatly simplify the construction, by completely avoiding the notion of good or good-enough position for almost invariant subsets.

There are two ingredients in our approach, to be found in Sections 3 and 4. (Section 2 recalls basic material about trees of cylinders, almost invariant sets, cross-connected components, and regular neighborhoods.)

The first ingredient is a general fact about almost invariant sets that are based on a given tree $T$. Consider any simplicial tree $T$ with an action of $G$. Any edge $e$ separates $T$ into two half-trees, and this defines almost invariant sets $Z_{e}$ and $Z_{e}^{*}$ (see Section 3a). The collection $\mathscr{B}(T)$ of almost invariant subsets based on $T$ is then defined by taking Boolean combinations of such sets $Z_{e}$.

Following Scott and Swarup, one defines cross-connected components of $\mathscr{B}(T)$ by using crossing of almost invariant sets. The set of cross-connected components 
is then endowed with a betweenness relation that allows one to construct a bipartite graph $R N(\mathscr{B}(T))$ associated to $\mathscr{B}(T)$. This is the regular neighborhood of $\mathscr{B}(T)$; see Definition 2.2.

Theorem 3.3. Let $G$ be a finitely generated group, and $T$ a tree with a minimal action of $G$. Assume that no two groups commensurable to edge stabilizers are contained in each other with infinite index.

Then the regular neighborhood $R N(\Re(T))$ is equivariantly isomorphic to a subdivision of $T_{c}$, the tree of cylinders of $T$ for the commensurability relation; in particular, $R N(\Re(T))$ is a tree.

The hypothesis about edge stabilizers holds in particular if all edge stabilizers of $T$ are $\mathrm{VPC}_{n}$ for a fixed $n$.

This theorem remains true if one enlarges $\mathscr{B}(T)$ to $\mathscr{B}(T) \cup \mathrm{QH}(T)$ by including almost invariant sets enclosed by quadratically hanging vertices of $T$. Geometrically, such a vertex is associated to a fiber bundle over a 2-dimensional orbifold $\mathbb{O}$. Any simple closed curve on $\mathbb{O}$ gives a way to blow up $T$ by creating new edges and therefore new almost invariant sets. These sets are in $\mathrm{QH}(T)$, as well as those associated to immersed curves on 0 . Under the same hypotheses as Theorem 3.3, we show in Theorem 3.11 that the regular neighborhood $R N(\Re(T) \cup \mathrm{QH}(T))$ also is a subdivision of $T_{c}$.

The second ingredient, specific to the $\mathrm{VPC}_{n}$ case, is due to (but not explicitly stated by) Scott and Swarup [2003]. We believe it is worth emphasizing this statement, as it gives a very useful description of almost invariant sets over $\mathrm{VPC}_{n}$ subgroups in terms of a JSJ splitting $T_{J}$. In plain words, it says that any almost invariant set over a $\mathrm{VPC}_{n}$ subgroup is either dual to a curve in a $\mathrm{QH}$ subgroup, or is a Boolean combination of almost invariant sets dual to half-trees of $T_{J}$.

Theorem 4.2 [Dunwoody and Swenson 2000; Scott and Swarup 2003]. Let $G$ and $T_{J}$ be as in Theorem 4.1 .

For any almost invariant subset $X$ over $a \mathrm{VPC}_{n}$ subgroup, the equivalence class $[X]$ belongs to $\mathscr{B}\left(T_{J}\right) \cup \mathrm{QH}\left(T_{J}\right)$.

Theorem 4.2 is essentially another take on the proof of Scott and Swarup's [2003, Theorem 8.2], and makes a crucial use of algebraic torus theorems of [Dunwoody and Swenson 2000; Dunwoody and Roller 1993]. We give a proof in Section 4.

Theorem 4.1 is a direct consequence of Theorems 4.2 and 3.11 .

\section{Preliminaries}

Let $G$ be a fixed finitely generated group, which, in Section 4, is finitely presented. 
2a. Trees. If $\Gamma$ is a graph, we denote by $V(\Gamma)$ its set of vertices and by $E(\Gamma)$ its set of (closed) nonoriented edges.

A tree always means a simplicial tree $T$ on which $G$ acts without inversions. Given a family $\mathscr{E}$ of subgroups of $G$, an $\mathscr{E}$-tree is a tree whose edge stabilizers belong to $\mathscr{E}$. We denote by $G_{v}$ or $G_{e}$ the stabilizer of a vertex $v$ or an edge $e$.

Given a subtree $A$, we denote by $\operatorname{pr}_{A}$ the projection onto $A$, mapping $x$ to the point of $A$ closest to $x$. If $A$ and $B$ are disjoint, or intersect in at most one point, then $\operatorname{pr}_{A}(B)$ is a single point, and we define the bridge between $A$ and $B$ as the segment joining $\operatorname{pr}_{A}(B)$ to $\operatorname{pr}_{B}(A)$.

A tree $T$ is nontrivial if there is no global fixed point, and minimal if there is no proper $G$-invariant subtree.

An element or a subgroup of $G$ is elliptic in $T$ if it has a global fixed point. An element that is not elliptic is hyperbolic. It has an axis on which it acts as a translation. If $T$ is minimal, then it is the union of all translation axes of elements of $G$. In particular, if $Y \subset T$ is a subtree, then any connected component of $T \backslash Y$ is unbounded.

A subgroup $A$ consisting only of elliptic elements fixes a point if it is finitely generated, a point or an end in general. If a finitely generated subgroup $A$ is not elliptic, there is a unique minimal $A$-invariant subtree.

A tree $T$ dominates a tree $T^{\prime}$ if there is an equivariant map $f: T \rightarrow T^{\prime}$. Equivalently, any subgroup that is elliptic in $T$ is also elliptic in $T^{\prime}$. Having the same elliptic subgroups is an equivalence relation on the set of trees, and the equivalence classes are called deformation spaces; see [Forester 2002; Guirardel and Levitt 2007] for details.

2b. Trees of cylinders. Two subgroups $A$ and $B$ of $G$ are commensurable if $A \cap B$ has finite index in both $A$ and $B$.

Definition 2.1. We fix a conjugacy-invariant family $\mathscr{E}$ of subgroups of $G$ such that

- any subgroup $A$ commensurable with some $B \in \mathscr{E}$ lies in $\mathscr{E}$, and

- if $A, B \in \mathscr{E}$ are such that $A \subset B$, then $[B: A]<\infty$.

An $\mathscr{E}$-tree is a tree whose edge stabilizers belong to $\mathscr{E}$.

For instance, $\mathscr{E}$ may consist of all subgroups of $G$ that are virtually $\mathbb{Z}^{n}$ for some fixed $n$, or all subgroups that are virtually polycyclic of Hirsch length exactly $n$.

In [Guirardel and Levitt 2008], we associated a tree of cylinders $T_{c}$ to any $\mathscr{E}$ tree $T$, as follows. Two (nonoriented) edges of $T$ are equivalent if their stabilizers are commensurable. A cylinder of $T$ is an equivalence class $Y$. We identify $Y$ with the union of its edges, which is a subtree of $T$.

Two distinct cylinders meet in at most one point. One can then define the tree of cylinders of $T$ as the tree $T_{c}$ dual to the covering of $T$ by its cylinders, as in 
[Guirardel 2004, Definition 4.8]. Formally, $T_{c}$ is the bipartite tree with vertex set $V\left(T_{c}\right)=V_{0}\left(T_{c}\right) \sqcup V_{1}\left(T_{c}\right)$ defined as follows:

(1) $V_{0}\left(T_{c}\right)$ is the set of vertices $x$ of $T$ belonging to (at least) two distinct cylinders;

(2) $V_{1}\left(T_{c}\right)$ is the set of cylinders $Y$ of $T$;

(3) there is an edge $\varepsilon=(x, Y)$ between $x \in V_{0}\left(T_{c}\right)$ and $Y \in V_{1}\left(T_{c}\right)$ if and only if $x$ (viewed as a vertex of $T$ ) belongs to $Y$ (viewed as a subtree of $T$ ).

Alternatively, one can define the boundary $\partial Y$ of a cylinder $Y$ as the set of vertices of $Y$ belonging to another cylinder, and obtain $T_{c}$ from $T$ by replacing each cylinder by the cone on its boundary.

All edges of a cylinder $Y$ have commensurable stabilizers, and we denote by $\mathscr{C} \subset \mathscr{E}$ the corresponding commensurability class. We sometimes view $V_{1}\left(T_{c}\right)$ as a set of commensurability classes.

2c. Almost invariant subsets. Given a subgroup $H \subset G$, consider the action of $H$ on $G$ by left multiplication. A subset $X \subset G$ is $H$-finite if it is contained in the union of finitely many $H$-orbits. Two subsets $X$ and $Y$ are equivalent if their symmetric difference $X+Y$ is $H$-finite. We denote by $[X]$ the equivalence class of $X$, and by $X^{*}$ the complement of $X$.

An $H$-almost invariant subset (or an almost invariant subset over $H$ ) is a subset $X \subset G$ that is invariant under the (left) action of $H$ and equivalent to the righttranslate $X s$ for all $s \in G$. An $H$-almost invariant subset $X$ is nontrivial if neither $X$ nor its complement $X^{*}$ is $H$-finite. Given $H<G$, the set of equivalence classes of $H$-almost invariant subsets is a Boolean algebra $\mathscr{P}_{H}$ for the usual operations.

If $H$ contains $H^{\prime}$ with finite index, then any $H$-almost invariant subset $X$ is also $H^{\prime}$-almost invariant. Furthermore, two sets $X$ and $Y$ are equivalent over $H^{\prime}$ if and only if they are equivalent over $H$. It follows that, given a commensurability class $\mathscr{C}$ of subgroups of $G$, the set of equivalence classes of almost invariant subsets over subgroups in $\mathscr{C}$ is a Boolean algebra $\mathscr{B}_{\mathfrak{C}}$.

Two almost invariant subsets $X$ over $H$ and $Y$ over $K$ are equivalent if their symmetric difference $X+Y$ is $H$-finite. By [Scott and Swarup 2003, Remark 2.9], this is a symmetric relation: $X+Y$ is $H$-finite if and only if it is $K$-finite. If $X$ and $Y$ are nontrivial, equivalence implies that $H$ and $K$ are commensurable.

The algebras $\mathscr{B}_{\mathscr{C}}$ are thus disjoint, except for the (trivial) equivalence classes of $\varnothing$ and $G$ that belong to every $\mathscr{B}_{\mathscr{C}}$. We denote by $\mathscr{B}$ the union of the algebras $\mathscr{B}_{\mathscr{C}}$. It is the set of equivalence classes of all almost invariant sets, but it is not a Boolean algebra in general. There is a natural action of $G$ on $\mathscr{B}$ induced by left translation (or conjugation).

2d. Cross-connected components and regular neighborhoods. Let $X$ be an $H$ almost invariant subset, and $Y$ a $K$-almost invariant subset. One says that $X$ 
crosses $Y$, or the pair $\left\{X, X^{*}\right\}$ crosses $\left\{Y, Y^{*}\right\}$, if none of the four sets $X^{(*)} \cap Y^{(*)}$ is $H$-finite (we denote by $X^{(*)} \cap Y^{(*)}$ the four possible intersections $X \cap Y, X^{*} \cap Y$, $X \cap Y^{*}$, and $\left.X^{*} \cap Y^{*}\right)$. By [Scott 1998], this is a symmetric relation. Note that $X$ and $Y$ do not cross if they are equivalent, and that crossing depends only on the equivalence classes of $X$ and $Y$. Following [Scott and Swarup 2003], we will say that $X^{(*)} \cap Y^{(*)}$ is small if it is $H$-finite (or equivalently $K$-finite).

Now let $\mathscr{X}$ be a subset of $\mathscr{B}$. Let $\overline{\mathscr{X}}$ be the set of nontrivial unordered pairs $\left\{[X],\left[X^{*}\right]\right\}$ for $[X] \in \mathscr{X}$. A cross-connected component $(\mathrm{CCC})$ of $\mathscr{X}$ is an equivalence class $C$ for the equivalence relation generated on $\overline{\mathscr{X}}$ by crossing. We often say that $X$, rather than $\left\{[X],\left[X^{*}\right]\right\}$, belongs to $C$, or represents $C$. We denote by $\mathscr{H}$ the set of cross-connected components of $\mathscr{X}$.

Given three distinct cross-connected components $C_{1}, C_{2}, C_{3}$, we say that $C_{2}$ is between $C_{1}$ and $C_{3}$ if there are representatives $X_{i}$ of $C_{i}$ satisfying $X_{1} \subset X_{2} \subset X_{3}$.

A star is a subset $\Sigma \subset \mathscr{H}$ containing at least two elements, and maximal for the property that, given $C, C^{\prime} \in \Sigma$, no $C^{\prime \prime} \in \mathscr{H}$ is between $C$ and $C^{\prime}$. We denote by $\mathscr{Y}$ the set of stars.

Definition 2.2. Let $\mathscr{L} \subset \mathscr{B}$ be a collection of almost invariant sets. Its regular neighborhood $R N(\mathscr{X})$ is the bipartite graph whose vertex set is $\mathscr{H} \sqcup \mathscr{Y}$ (a vertex is either a cross-connected component or a star), and whose edges are pairs $(C, \Sigma) \in \mathscr{H} \times \mathscr{Y}$ with $C \in \Sigma$. If $\mathscr{L}$ is $G$-invariant, then $G$ acts on $R N(\mathscr{X})$.

This definition is motivated by the following remark, whose proof we leave to the reader.

Remark 2.3. Let $T$ be any simplicial tree. Suppose that $\mathscr{H} \subset T$ meets any closed edge in a nonempty finite set. Define betweenness in $\mathscr{H}$ by $C_{2} \in\left[C_{1}, C_{3}\right] \subset T$. Then the bipartite graph defined as above is isomorphic to a subdivision of $T$.

In the situation of Scott and Swarup [2003], a main result is that $R N(\mathscr{C})$ is a tree. We will reprove this fact by identifying $R N(\mathscr{X})$ with a subdivision of the tree of cylinders.

\section{Regular neighborhoods as trees of cylinders}

Now we fix a family $\mathscr{E}$ as in Definition 2.1. It is stable under commensurability, and a group of $\mathscr{E}$ cannot contain another with infinite index. Let $T$ be an $\mathscr{E}$-tree.

In Section 3a, we define the set $\mathscr{B}(T)$ of almost invariant sets based on $T$, and we state the main result, Theorem 3.3: The regular neighborhood $R N(\mathscr{B}(T))$ of $\mathscr{B}(T)$ is up to subdivision the tree of cylinders $T_{c}$. In Section $3 \mathrm{~b}$, we represent elements of $\mathscr{B}(T)$ by special subforests of $T$. We then study the cross-connected components of $\mathscr{B}(T)$. We prove Theorem 3.3 in Section $3 \mathrm{~d}$ by constructing a map $\Phi$ from the set of cross-connected components to $T_{c}$. In Section $3 \mathrm{e}$ we generalize Theorem 3.3 to 
Theorem 3.11 by including almost invariant sets enclosed by quadratically hanging vertices of $T$.

3a. Almost invariant sets based on a tree. We fix a basepoint $v_{0} \in V(T)$. If $e$ is an edge of $T$, we denote by $\stackrel{e}{e}$ the open edge. Let $T_{e}$ and $T_{e}^{*}$ be the connected components of $T \backslash \grave{e}$. The set of $g \in G$ such that $g v_{0} \in T_{e}$ (respectively $g v_{0} \in T_{e}^{*}$ ) is an almost invariant set $Z_{e}$ (respectively $Z_{e}^{*}$ ) over $G_{e}$. Up to equivalence, it is independent of $v_{0}$. When we need to distinguish between $Z_{e}$ and $Z_{e}^{*}$, we orient $e$ and declare that the terminal vertex of $e$ belongs to $T_{e}$.

Now consider a cylinder $Y \subset T$ and the corresponding commensurability class $\mathscr{b}$. Any Boolean combination of the $Z_{e}$ for $e \in E(Y)$ is an almost invariant set over some subgroup $H \in \mathscr{C}$.

Definition 3.1. Given a cylinder $Y$, associated to a commensurability class $\mathscr{C}$, the Boolean algebra of almost invariant subsets based on $Y$ is the subalgebra $\mathscr{B}_{\mathscr{C}}(T)$ of $\mathscr{B}_{\mathscr{C}}$ generated by the classes $\left[Z_{e}\right]$ for $e \in E(Y)$.

The set of almost invariant subsets based on $T$ is the union $\mathscr{B}(T)=\bigcup_{\mathscr{C}} \mathscr{B}_{\mathscr{C}}(T)$, a subset of $\mathscr{B}=\bigcup_{\mathscr{C}} \mathscr{B}_{\mathscr{C}}$; just like $\mathscr{B}$, it is a union of Boolean algebras but not itself a Boolean algebra.

Proposition 3.2. Let $T$ and $T^{\prime}$ be minimal $\mathscr{E}$-trees. Then $\mathscr{B}(T)=\mathscr{B}\left(T^{\prime}\right)$ if and only if $T$ and $T^{\prime}$ belong to the same deformation space.

More precisely, $T$ dominates $T^{\prime}$ if and only if $\mathscr{B}\left(T^{\prime}\right) \subset \mathscr{B}(T)$.

Proof. Suppose $T$ dominates $T^{\prime}$. After subdividing $T$ (this does not change $\mathscr{B}(T)$ ), we may assume that there is an equivariant map $f: T \rightarrow T^{\prime}$ sending every edge to a vertex or an edge. We claim that, given $e^{\prime} \in E\left(T^{\prime}\right)$, there are only finitely many edges $e_{i} \in E(T)$ such that $f\left(e_{i}\right)=e^{\prime}$. To see this, we may restrict to a $G$-orbit of edges of $T$, since there are finitely many such orbits. If $e$ and ge both map onto $e^{\prime}$, then $g \in G_{e^{\prime}}$. Because of the hypotheses on $\mathscr{E}$, the stabilizer $G_{e}$ is contained in $G_{e^{\prime}}$ with finite index. The claim follows.

Choose basepoints $v \in T$ and $v^{\prime}=f(v) \in T^{\prime}$. Then $Z_{e^{\prime}}$ (defined using $v^{\prime}$ ) is a Boolean combination of the sets $Z_{e_{i}}$ (defined using $v$ ), so $\mathscr{B}\left(T^{\prime}\right) \subset \mathscr{B}(T)$.

Conversely, assume $\mathscr{B}\left(T^{\prime}\right) \subset \mathscr{B}(T)$. Let $K \subset G$ be a subgroup elliptic in $T$. We show that it is also elliptic in $T^{\prime}$.

If not, we can find an edge $e^{\prime}=\left[v^{\prime}, w^{\prime}\right] \subset T^{\prime}$, and sequences $g_{n} \in G$ and $k_{n} \in K$, such that the sequences $g_{n} v^{\prime}$ and $g_{n} k_{n} v^{\prime}$ have no bounded subsequence, and $e^{\prime} \subset\left[g_{n} v^{\prime}, g_{n} k_{n} v^{\prime}\right]$ for all $n$. (If $K$ contains a hyperbolic element $k$, we choose $e^{\prime}$ on its axis, and we define $g_{n}=k^{-n}$ and $k_{n}=k^{2 n}$; if $K$ fixes an end $\omega$, we want $g_{n}^{-1} e^{\prime} \subset\left[v^{\prime}, k_{n} v^{\prime}\right]$, so we choose $e^{\prime}$ and $g_{n}$ such that all edges $g_{n}^{-1} e^{\prime}$ are contained on a ray $\rho$ going out to $\omega$, and then we choose $k_{n}$.) Defining $Z_{e^{\prime}}$ using the vertex $v^{\prime}$ and a suitable orientation of $e^{\prime}$, we have $g_{n} \in Z_{e^{\prime}}$ and $g_{n} k_{n} \notin Z_{e^{\prime}}$. 
Using a vertex of $T$ fixed by $K$ to define the almost invariant sets $Z_{e}$, we see that any element of $\mathscr{B}(T)$ is represented by an almost invariant set $X$ satisfying $X K=X$. In particular, since $\mathscr{B}\left(T^{\prime}\right) \subset \mathscr{B}(T)$, there exist finite sets $F_{1}$ and $F_{2}$ such that $Z=\left(Z_{e^{\prime}} \backslash G_{e^{\prime}} F_{1}\right) \cup G_{e^{\prime}} F_{2}$ is $K$-invariant on the right. For every $n$, one has $g_{n} k_{n} \in G_{e^{\prime}} F_{2}$ (if $g_{n}, g_{n} k_{n} \in Z$ ) or $g_{n} \in G_{e^{\prime}} F_{1}$ (if not), so one of the sequences $g_{n} k_{n} v^{\prime}$ or $g_{n} v^{\prime}$ has a bounded subsequence (because $G_{e^{\prime}}$ is elliptic), a contradiction.

Remark. The only fact used in the proof is that no edge stabilizer of $T$ has infinite index in an edge stabilizer of $T^{\prime}$.

Theorem 3.3. Let $T$ be a minimal $\mathscr{E}$-tree, with $\mathscr{E}$ as in Definition 2.1 , and $T_{c}$ its tree of cylinders for the commensurability relation. Let $\mathscr{X}=\mathscr{B}(T)$ be the set of almost invariant subsets based on $T$.

Then $R N(\mathscr{C})$ is equivariantly isomorphic to a subdivision of $T_{c}$.

By Proposition 3.2, and [Guirardel and Levitt 2008, Theorem 1], $R N(\mathscr{C})$ and $T_{c}$ only depend on the deformation space of $T$.

To prove the version of Theorem 3.3 stated in the introduction, one takes $\mathscr{E}$ to be the family of subgroups commensurable to an edge stabilizer of $T$.

The theorem will be proved in the next three subsections. We always fix a base vertex $v_{0} \in T$.

3b. Special forests. Let $S$ and $S^{\prime}$ be subsets of $V(T)$. We say that $S$ and $S^{\prime}$ are equivalent if their symmetric difference is finite; we say $S$ is trivial if it is equivalent to $\varnothing$ or $V(T)$.

The coboundary $\delta S$ is the set of edges having one endpoint in $S$ and one in $S^{*}$ (the complement of $S$ in $V(T)$ ). We shall be interested in sets $S$ with finite coboundary. Since $\delta\left(S \cap S^{\prime}\right) \subset \delta S \cup \delta S^{\prime}$, they form a Boolean algebra.

We also view such an $S$ as a subforest of $T$, by including all edges whose endpoints are both in $S$; we can then consider the (connected) components of $S$. The set of edges of $T$ is partitioned into edges in $S$, edges in $S^{*}$, and edges in $\delta S=\delta S^{*}$. Note that $S$ is equivalent to the set of endpoints of its edges. In particular, $S$ is finite (as a set of vertices) if and only if it contains finitely many edges.

We say that $S$ is a special forest based on a cylinder $Y$ if $\delta S=\left\{e_{1}, \ldots, e_{n}\right\}$ is finite and contained in $Y$. If nonempty, $S$ contains at least one vertex of $Y$. Each component of $S$ (viewed as a subforest) is a component of $T \backslash\left\{\stackrel{\circ}{e}_{1}, \ldots, \stackrel{\bullet}{e}_{n}\right\}$, and $S^{*}$ is the union of the other components of $T \backslash\left\{\stackrel{e}{e}_{1}, \ldots, \stackrel{\circ}{e}_{n}\right\}$.

We define $\mathscr{B}_{Y}$ as the Boolean algebra of equivalence classes of special forests based on $Y$.

Given a special forest $S$ based on $Y$, we define $X_{S}=\left\{g \mid g v_{0} \in S\right\}$. It is an almost invariant set over $H=\bigcap_{e \in \delta S} G_{e}$, a subgroup of $G$ belonging to the commensurability class $\mathscr{C}$ associated to $Y$; we denote its equivalence class by $\left[X_{S}\right]$. Every element of $\mathscr{B}(T)$ may be represented in this form. More precisely: 
Lemma 3.4. Let $Y$ be a cylinder associated to a commensurability class $\mathscr{\complement}$. Then the map $S \mapsto\left[X_{S}\right]$ induces an isomorphism of Boolean algebras between $\mathscr{B}_{Y}$ and $\mathscr{B}_{\mathscr{C}}(T)$.

Proof. It is easy to check that $S \mapsto\left[X_{S}\right]$ is a morphism of Boolean algebras. It is onto because the set $T_{e}$ used to define the almost invariant set $Z_{e}$ is a special forest (based on the cylinder containing $e$ ). It remains to determine the "kernel", namely to show that $X_{S}$ is $H$-finite if and only if $S$ is finite (where $H$ denotes any group in $\mathscr{C})$.

First suppose that $S$ is finite. Then $S$ is contained in $Y$ since it contains any connected component of $T \backslash Y$ that it intersects. Since $\delta S$ is finite, no vertex $x$ of $S$ has infinite valence in $T$. In particular, for each vertex $x \in S$, the group $G_{x}$ is commensurable with $H$. It follows that $\left\{g \in G \mid g . v_{0}=x\right\}$ is $H$-finite, and $X_{S}$ is $H$-finite.

If $S$ is infinite, one of its components is infinite, and by minimality of $T$ there exists a hyperbolic element $g \in G$ such that $g^{n} v_{0} \in S$ for all $n \geq 0$. Thus $g^{n} \in X_{S}$ for $n \geq 0$. If $X_{S}$ is $H$-finite, one can find a sequence $n_{i}$ going to infinity, and $h_{i} \in H$, such that $g^{n_{i}}=h_{i} g^{n_{0}}$. Since $H$ is elliptic in $T$, the sequence $h_{i} g^{n_{0}} v_{0}$ is bounded, a contradiction.

Lemma 3.5. Let $S$ and $S^{\prime}$ be special forests.

(1) If $S$ and $S^{\prime}$ are infinite and based on distinct cylinders, and if $S \cap S^{\prime}$ is finite, then $S \cap S^{\prime}=\varnothing$.

(2) If $X_{S}$ crosses $X_{S^{\prime}}$, then $S$ and $S^{\prime}$ are based on the same cylinder.

(3) $X_{S} \cap X_{S^{\prime}}$ is small if and only if $S \cap S^{\prime}$ is finite.

Proof. For part (1), assume that $S$ and $S^{\prime}$ are infinite and based on $Y \neq Y^{\prime}$, and that $S \cap S^{\prime}$ is finite. Let $\left[u, u^{\prime}\right]$ be the bridge between $Y$ and $Y^{\prime}$ (with $u=u^{\prime}$ if $Y$ and $Y^{\prime}$ intersect in a point). Since $u$ and $u^{\prime}$ lie in more than one cylinder, they have infinite valence in $T$.

Assume first that $u \in S$. Then $S$ contains all components of $T \backslash\{u\}$, except finitely many of them (which intersect $Y$ ). In particular, $S$ contains $Y^{\prime}$. If $S^{\prime}$ contains $u^{\prime}$, it contains $u$ by the same argument, and $S \cap S^{\prime}$ contains infinitely many edges incident on $u$, a contradiction. If $S^{\prime}$ does not contain $u^{\prime}$, it is contained in $S$, also a contradiction.

We may therefore assume $u \notin S$ and $u^{\prime} \notin S^{\prime}$. It follows that $S$ (respectively $S^{\prime}$ ) is contained in the union of the components of $T \backslash\{u\}$ (respectively $T \backslash\left\{u^{\prime}\right\}$ ) which intersect $Y$ (respectively $Y^{\prime}$ ), so $S$ and $S^{\prime}$ are disjoint.

Part (2) is a consequence of [Scott and Swarup 2003, Proposition 13.5], but here is a direct argument. Assume that $S$ and $S^{\prime}$ are based on $Y \neq Y^{\prime}$, and let $\left[u, u^{\prime}\right]$ 
be as above. Up to replacing $S$ and $S^{\prime}$ by their complements, we have $u \notin S$ and $u^{\prime} \notin S^{\prime}$. The argument above shows that $S \cap S^{\prime}=\varnothing$, so $X_{S}$ does not cross $X_{S^{\prime}}$.

For part (3), first suppose that $S \cap S^{\prime}$ is finite. If, say, $S$ is finite, then $X_{S}$ is $H$-finite by Lemma 3.4, so $X_{S} \cap X_{S^{\prime}}$ is small. Assume therefore that $S$ and $S^{\prime}$ are infinite. If they are based on distinct cylinders, then $X_{S} \cap X_{S^{\prime}}=\varnothing$ by part (1). If they are based on the same cylinder, then $S \cap S^{\prime}$ is itself a finite special forest, so $X_{S} \cap X_{S^{\prime}}=X_{S \cap S^{\prime}}$ is small by Lemma 3.4. Conversely, if $S \cap S^{\prime}$ is infinite, one shows that $X_{S} \cap X_{S^{\prime}}$ is not $H$-finite as in the proof of Lemma 3.4, using $g$ such that $g^{n} v \in S \cap S^{\prime}$ for all $n \geq 0$.

Remark 3.6. If $S$ and $S^{\prime}$ are infinite and $X_{S} \cap X_{S^{\prime}}$ is small, then $S$ and $S^{\prime}$ are equivalent to disjoint special forests. This follows from the lemma if they are based on distinct cylinders. If not, one replaces $S^{\prime}$ by $S^{\prime} \cap S^{*}$.

3c. Peripheral cross-connected components. Theorem 3.3 is trivial if $T$ is a line, so we can assume that each vertex of $T$ has valence at least 3 (we now allow $G$ to act with inversions). We need to understand cross-connected components. By Lemma 3.5(2), every such component is based on a cylinder, so we focus on a given $Y$. We first define peripheral special forests and almost invariant sets.

Recall that $\partial Y$ is the set of vertices of $Y$ that belong to another cylinder. Let $v \in \partial Y$ be a vertex whose valence in $Y$ is finite. Let $e_{1}, \ldots, e_{n}$ be the edges of $Y$ containing $v$, oriented towards $v$. Let $S_{v, Y}=T_{e_{1}} \cap \cdots \cap T_{e_{n}}$ (recall that $T_{e}$ denotes the component of $T \backslash \stackrel{e}{e}$ containing the terminal point of $e$ ). It is a subtree satisfying $S_{v, Y} \cap Y=\{v\}$, with coboundary $\delta S_{v, Y}=\left\{e_{1}, \ldots, e_{n}\right\}$. We say that $S_{v, Y}$, and any special forest equivalent to it, is peripheral (but $S_{v, Y}^{*}$ is not peripheral in general).

We denote by $X_{v, Y}$ the almost invariant set corresponding to $S_{v, Y}$, and we say that $X$ is peripheral if it is equivalent to some $X_{v, Y}$. Both $S_{v, Y}$ and $S_{v, Y}^{*}$ are infinite, so $X_{v, Y}$ is nontrivial by Lemma 3.4.

We claim that $C_{v, Y}=\left\{\left\{\left[X_{v, Y}\right],\left[X_{v, Y}^{*}\right]\right\}\right\}$ is a complete cross-connected component of $\mathscr{B}(T)$, called a peripheral CCC. Indeed, assume that $X_{v, Y}$ crosses some $X_{S}$. Then $S$ is based on $Y$ by Lemma 3.5, but since $S_{v, Y}$ contains no edge of $Y$, it is contained in $S_{X}$ or $S_{X}^{*}$, which prevents crossing.

Note that if $C_{v, Y}=C_{v^{\prime}, Y^{\prime}}$, then $Y=Y^{\prime}$ (because an $H$-almost invariant subset determines the commensurability class of $H$ ), and $v=v^{\prime}$ except when $Y$ is a single edge $v v^{\prime}$, in which case $X_{v, Y}=X_{v^{\prime}, Y}^{*}$.

Lemma 3.7. Let $Y$ be a cylinder. There is at most one nonperipheral crossconnected component $C_{Y}$ based on $Y$. There is exactly one if and only if $|\partial Y| \neq 2,3$.

Proof. The proof is in three parts.

We first claim that, given any infinite connected nonperipheral special forest $S$ based on $Y$, there is an edge $e \subset S \cap Y$ such that both connected components of $S \backslash\{\mathfrak{e}\}$ are infinite. 
Assume there is no such $e$. Then $S \cap Y$ is locally finite: Given $v \in S$, all but finitely many components of $S \backslash\{v\}$ are infinite, so infinitely many edges incident on $v$ satisfy the claim if $v$ has infinite valence in $S \cap Y$.

Since $S$ is infinite and nonperipheral, $S \cap Y$ is not reduced to a single point. We orient every edge $e$ of $S \cap Y$ so that $S \cap T_{e}$ is infinite and $S \cap T_{e}^{*}$ is finite. If a vertex $v$ of $S \cap Y$ is terminal in every edge of $S \cap Y$ that contains it, $S$ is peripheral. We may therefore find an infinite ray $\rho \subset S \cap Y$ consisting of positively oriented edges. Since every vertex of $T$ has valence $\geq 3$, every vertex of $\rho$ is the projection onto $\rho$ of an edge of $\delta S$, contradicting the finiteness of $\delta S$. This proves the claim.

Secondly, to show that there is at most one nonperipheral cross-connected component, we fix two nontrivial forests $S$ and $S^{\prime}$ based on $Y$, and we show that $X_{S}$ and $X_{S^{\prime}}$ are in the same CCC if they do not belong to peripheral CCCs. We can assume that $X_{S} \cap X_{S^{\prime}}$ is small, and by Remark 3.6 that $S \cap S^{\prime}$ is empty. We may also assume that every component of $S$ and $S^{\prime}$ is infinite.

Since $S$ is not peripheral, it contains two disjoint infinite special forests $S_{1}$ and $S_{2}$ based on $Y$ : This is clear if $S$ has several components, and follows from the claim otherwise. Construct $S_{1}^{\prime}$ and $S_{2}^{\prime}$ similarly. Then $X_{S_{1}} \cup X_{S_{1}^{\prime}}$ crosses both $X_{S}$ and $X_{S^{\prime}}$, so $X_{S}$ and $X_{S^{\prime}}$ are in the same cross-connected component.

Finally, we discuss the existence of $C_{Y}$. If $|\partial Y| \geq 4$, choose $v_{1}, \ldots, v_{4} \in \partial Y$, and consider edges $e_{1}, e_{2}, e_{3}$ of $Y$ such that each $v_{i}$ belongs to a different component $S_{i}$ of $T \backslash\left\{\stackrel{\circ}{e}_{1}, \stackrel{\circ}{e}_{2}, \stackrel{\circ}{e}_{3}\right\}$. These components are infinite because $v_{i} \in \partial Y$, and $X_{S_{1} \cup S_{2}}$ belongs to a nonperipheral CCC.

If $\partial Y$ is empty, then $Y=T$ and existence is clear. If $\partial Y$ is nonempty, minimality of $T$ implies that $Y$ is the convex hull of $\partial Y$ (replacing every cylinder by the convex hull of its boundary yields an invariant subtree). From this we deduce that $|\partial Y| \neq 1$, and every CCC based on $Y$ is peripheral if $|\partial Y|$ equals 2 or 3 . There is one peripheral CCC if $|\partial Y|=2$ (that is, $Y$ is a single edge) and three if $|\partial Y|=3$.

Remark 3.8. The proof shows that, if $|\partial Y| \geq 4$, then for all $u \neq v$ in $\partial Y$, the nonperipheral CCC is represented by a special forest $S$ such that $u \in S$ and $v \in S^{*}$.

3d. Proof of Theorem 3.3. From now on we assume that $T$ has more than one cylinder; otherwise there is exactly one cross-connected component, and both $R N(\mathscr{X})$ and $T_{c}$ are points.

It will be helpful to distinguish between a cylinder $Y \subset T$ or a point $\eta \in \partial Y$, and the corresponding vertex of $T_{c}$. We therefore denote by $Y_{c}$ or $\eta_{c}$ the vertex of $T_{c}$ corresponding to $Y$ or $\eta$.

Recall that $\mathscr{H}$ denotes the set of cross-connected components of $\mathscr{X}=\mathscr{B}(T)$. Consider the map $\Phi: \mathscr{H} \rightarrow T_{c}$ defined as follows:

- If $C=C_{Y}$ is a nonperipheral CCC, then $\Phi(C)=Y_{c} \in V_{1}\left(T_{c}\right)$. 
- If $C=C_{v, Y}$ is peripheral, and \# $Y \geq 3$, then $\Phi(C)$ is the midpoint of the edge $\varepsilon=\left(v_{c}, Y_{c}\right)$ of $T_{c}$.

- If \# $Y Y=2$, and $C$ is the peripheral CCC based on $Y$, then $\Phi(C)=Y_{c}$.

In all cases, the distance between $\Phi(C)$ and $Y_{c}$ is at most $1 / 2$. If $C$ is peripheral, $\Phi(C)$ has valence 2 in $T_{c}$.

Clearly, $\Phi$ is one-to-one. By Remark 2.3, it now suffices to show that the image of $\Phi$ meets every closed edge, and $\Phi$ preserves betweenness: For $C_{1}, C_{2}, C_{3} \in \mathscr{H}$, $C_{2}$ is between $C_{1}$ and $C_{3}$ if and only if $\Phi\left(C_{2}\right) \in\left[\Phi\left(C_{1}\right), \Phi\left(C_{3}\right)\right]$.

The first fact is clear because $\Phi(\mathscr{H})$ contains all vertices $Y_{c} \in V_{1}\left(T_{c}\right)$ with $|\partial Y| \neq 3$ and the three points at distance $1 / 2$ from $Y_{c}$ if $|\partial Y|=3$. To control betweenness, we need a couple of technical lemmas.

If $S$ is a nontrivial special forest, we denote by [[S] the cross-connected component represented by the almost invariant set $X_{S}$.

Let $Y \subset T$ be a cylinder. We denote by $\operatorname{pr}_{Y}: T \rightarrow Y$ the projection. If $Y^{\prime}$ is another cylinder, then $\operatorname{pr}_{Y}\left(Y^{\prime}\right)$ is a single point. This point belongs to two cylinders and hence defines a vertex of $V_{0}\left(T_{c}\right)$ that is at distance 1 from $Y_{c}$ on the segment of $T_{c}$ joining $Y_{c}$ to $Y_{c}^{\prime}$.

Let $Y$ be a cylinder with $|\partial Y| \geq 4$. For each nontrivial special forest $S^{\prime}$ that is either based on some $Y^{\prime} \neq Y$, or based on $Y$ and peripheral, we define a point $\eta_{Y}\left(S^{\prime}\right) \in Y \subset T$ as follows. If $S^{\prime}$ is based on $Y^{\prime} \neq Y$, we define $\eta_{Y}\left(S^{\prime}\right)$ to be $\operatorname{pr}_{Y}\left(Y^{\prime}\right)$. If $S^{\prime}$ is equivalent to some $S_{v, Y}$, we define $\eta_{Y}\left(S^{\prime}\right)=v$; note that in this case $\eta_{Y}\left(S^{*}\right)$ is not defined.

Lemma 3.9. Let $Y$ be a cylinder with $|\partial Y| \geq 4$. Consider two nontrivial special forests $S, S^{\prime}$ with $\llbracket S^{\prime} \rrbracket \neq C_{Y}$ and $\llbracket S \rrbracket=C_{Y}$, and assume $S^{\prime} \subset S$.

Then $\eta=\eta_{Y}\left(S^{\prime}\right) \in Y$ is defined, $\eta \in S$, and $S^{\prime}$ contains an equivalent subforest $S^{\prime \prime}$ with $S^{\prime \prime} \subset \operatorname{pr}_{Y}^{-1}(\{\eta\}) \subset S$.

Moreover, $\left.\left.\Phi\left(\llbracket S^{\prime}\right]\right]\right)$ lies in the connected component of $T_{c} \backslash\left\{Y_{c}\right\}$ containing $\eta_{c}$.

Proof. Let $Y^{\prime}$ be the cylinder on which $S^{\prime}$ is based.

If $Y^{\prime}=Y$, then $S^{\prime *}$ is not peripheral, so $S^{\prime}$ is peripheral. Thus $\eta$ is defined, and $S^{\prime}$ is equivalent to its subforest $S^{\prime \prime}=S_{Y, \eta}$. Then $S^{\prime \prime}=\operatorname{pr}_{Y}^{-1}(\{\eta\}) \subset S$. In this case $\Phi\left(\llbracket S^{\prime} \rrbracket\right)$ is the midpoint of the edge $\left(\eta_{c}, Y_{c}\right)$ of $T_{c}$.

Assume that $Y^{\prime} \neq Y$. Then $\eta=\operatorname{pr}_{Y}\left(Y^{\prime}\right) \in S$; otherwise $Y^{\prime}$ would be disjoint from $S$ and hence from $S^{\prime}$, a contradiction. It follows that $\operatorname{pr}_{Y}^{-1}(\{\eta\}) \subset S$. If $\eta \in S^{\prime}$, then $S^{\prime}$ contains the complement of $\operatorname{pr}_{Y}^{-1}(\{\eta\})$, so $S=T$, a contradiction. Thus $\eta \notin S^{\prime}$ and therefore $S^{\prime} \subset \operatorname{pr}_{Y}^{-1}(\{\eta\})$. The "moreover" is clear in this case since $\eta_{c}$ is between $Y_{c}$ and $Y_{c}^{\prime}$, and $\Phi\left(\left[\left[S^{\prime}\right]\right]\right)$ is at distance $\leq 1 / 2$ from $Y_{c}^{\prime}$.

Lemma 3.10. Let $S=S_{Y, u}$ be peripheral, and let $S^{\prime}$ be a nontrivial special forest with $\left[\left[S^{\prime} \rrbracket \neq \llbracket S\right]\right.$. Recall that $u_{c}$ is the vertex of $T_{c}$ associated to $u$. 
(1) If $S^{\prime} \subset S$, then $\left.\Phi\left(\llbracket S^{\prime}\right]\right)$ belongs to the component of $T_{c} \backslash\{\Phi(\llbracket S \rrbracket)\}$ that contains $u_{c}$.

(2) If $S \subset S^{\prime}$, then $\left.\left.\Phi\left(\llbracket S^{\prime}\right]\right]\right)$ belongs to the component of $\left.\left.T_{c} \backslash\{\Phi(\llbracket S]]\right)\right\}$ that does not contain $u_{c}$.

Proof. If $S^{\prime} \subset S$, then $S^{\prime}$ is based on some $Y^{\prime} \neq Y$. Since $S^{\prime} \subset S=\operatorname{pr}_{Y}^{-1}(\{u\})$, we have $Y^{\prime} \subset \operatorname{pr}_{Y}^{-1}(\{u\})$ and $u_{c}$ is between $Y_{c}$ and $Y_{c}^{\prime}$ in $T_{c}$. The result follows since $\Phi(\llbracket S]])$ is $1 / 2$-close to $Y_{c}$ and $\Phi\left(\left[\left[S^{\prime} \rrbracket\right)\right.\right.$ is $1 / 2$-close to $Y_{c}^{\prime}$.

If $S \subset S^{\prime}$ and $Y \neq Y^{\prime}$, we have $\operatorname{pr}_{Y}\left(Y^{\prime}\right) \neq u$ because $S^{\prime} \neq T$, and the lemma follows. If $Y=Y^{\prime}$, the lemma is immediate.

We can now show that $\Phi$ preserves betweenness. Consider three distinct crossconnected components $C_{1}, C_{2}, C_{3} \in \mathcal{H}$. Let $Y_{2}$ be the cylinder on which $C_{2}$ is based. Note that $\left|\partial Y_{2}\right| \geq 4$ if $C_{2}$ is nonperipheral.

First assume that $C_{2}$ is between $C_{1}$ and $C_{3}$. By definition, there exist almost invariant subsets $X_{i}$ representing $C_{i}$ such that $X_{1} \subset X_{2} \subset X_{3}$. By Lemma 3.4, one can find special forests $S_{i}$ with $\left[X_{S_{i}}\right]=\left[X_{i}\right]$. By Remark 3.6, since the $C_{i}$ are distinct, one can assume $S_{1} \subset S_{2} \subset S_{3}$ (if necessary, replace $S_{2}$ by $S_{2} \cap S_{3}$, and then $S_{1}$ by $\left.S_{1} \cap S_{2} \cap S_{3}\right)$.

If $S_{2}$ is peripheral, $\Phi\left(C_{1}\right)$ and $\Phi\left(C_{3}\right)$ are in distinct components of $T_{c} \backslash\left\{\Phi\left(C_{2}\right)\right\}$ by Lemma 3.10, so $\Phi\left(C_{2}\right) \in\left[\Phi\left(C_{1}\right), \Phi\left(C_{3}\right)\right]$. If $S_{2}^{*}$ is peripheral, we apply the same argument using $S_{3}^{*} \subset S_{2}^{*} \subset S_{1}^{*}$.

Assume therefore that $C_{2}$ is nonperipheral. Lemma 3.9 implies that the points $\eta_{1}=\eta_{Y_{2}}\left(S_{1}\right)$ and $\eta_{3}=\eta_{Y_{2}}\left(S_{3}^{*}\right)$ are defined, and $\eta_{1} \in S_{2}$ and $\eta_{3} \in S_{2}^{*}$. In particular, we have $\eta_{1} \neq \eta_{3}$. By the "moreover", we get $\Phi\left(C_{2}\right) \in\left[\Phi\left(C_{1}\right), \Phi\left(C_{3}\right)\right]$ since $\Phi\left(C_{2}\right)=\left(Y_{2}\right)_{c}$.

Now assume that $C_{2}$ is not between $C_{1}$ and $C_{3}$, and choose $S_{i}$ with $\llbracket S_{i} \rrbracket=C_{i}$. By Remark 3.6, we may assume that for each $i \in\{1,3\}$ some inclusion $S_{i}^{(*)} \subset S_{2}^{(*)}$ holds. Since $C_{2}$ is not between $C_{1}$ and $C_{3}$, we may assume after changing $S_{i}$ to $S_{i}^{*}$ if needed that $S_{1} \subset S_{2}$ and $S_{3} \subset S_{2}$.

If $S_{2}$ or $S_{2}^{*}$ is peripheral, Lemma 3.10 implies that $\Phi\left(C_{1}\right)$ and $\Phi\left(C_{3}\right)$ lie in the same connected component of $T_{c} \backslash\left\{\Phi\left(C_{2}\right)\right\}$, so $\Phi\left(C_{2}\right)$ is not between $\Phi\left(C_{1}\right)$ and $\Phi\left(C_{3}\right)$.

Assume therefore that $C_{2}$ is nonperipheral. Lemma 3.9 says that the points $\eta_{1}=\eta_{Y_{2}}\left(S_{1}\right)$ and $\eta_{3}=\eta_{Y_{2}}\left(S_{3}\right)$ are defined, and we may assume $S_{i} \subset \operatorname{pr}_{Y_{2}}^{-1}\left(\left\{\eta_{i}\right\}\right)$. If $\eta_{1}=\eta_{3}$, then $\Phi\left(C_{2}\right)$ does not lie between $\Phi\left(C_{1}\right)$ and $\Phi\left(C_{3}\right)$ by the "moreover" of Lemma 3.9. If $\eta_{1} \neq \eta_{3}$, consider $\tilde{S}_{2}$ with $\left[\left[\tilde{S}_{2}\right]\right]=C_{2}$ such that $\eta_{1} \in \tilde{S}_{2}$ and $\eta_{3} \in \tilde{S}_{2}^{*}$ (it exists by Remark 3.8). Then $S_{1} \subset \operatorname{pr}_{Y_{2}}^{-1}\left(\eta_{1}\right) \subset \tilde{S}_{2}$ and $S_{3} \subset \operatorname{pr}_{Y_{2}}^{-1}\left(\eta_{3}\right) \subset \tilde{S}_{2}^{*}$, so $C_{2}$ lies between $C_{1}$ and $C_{3}$, a contradiction.

This ends the proof of Theorem 3.3. 
3e. Quadratically hanging vertices. We say that a vertex stabilizer $G_{v}$ of $T$ is a $Q H$-subgroup if there is an exact sequence $1 \rightarrow F \rightarrow G_{v} \stackrel{\pi}{\rightarrow} \Sigma \rightarrow 1$, where $\Sigma=\pi_{1}(\mathcal{O})$ is a hyperbolic 2-orbifold group and every incident edge group $G_{e}$ is peripheral: It is contained with finite index in the preimage by $\pi$ of a boundary subgroup $B=\pi_{1}(C)$, with $C$ a boundary component of $\mathrm{O}$. We say that $v$ is a $Q H$-vertex of $T$.

We now define almost invariant sets based on $v$. They will be included in our description of the regular neighborhood.

We view $\Sigma$ as a convex cocompact Fuchsian group acting on $\mathbb{H}^{2}$. Let $\bar{H}$ be any nonperipheral maximal two-ended subgroup of $\Sigma$ (represented by an immersed curve or 1-suborbifold). Let $\gamma$ be the geodesic invariant by $\bar{H}$. It separates $\mathbb{U}^{2}$ into two half-spaces $P^{ \pm}$, which may be interchanged by certain elements of $\bar{H}$.

Let $\bar{H}_{0}$ be the stabilizer of $P^{+}$, which has index at most 2 in $\bar{H}$, and let $x_{0}$ be a basepoint. We define an $\bar{H}_{0}$-almost invariant set $\bar{X} \subset \Sigma$ as the set of $g \in \Sigma$ such that $g x_{0} \in P^{+}$. (If $\bar{H}$ is the fundamental group of a two-sided simple closed curve on $\mathcal{O}$, there is a one-edge splitting of $\Sigma$ over $\bar{H}$, and $\bar{X}$ is a $Z_{e}$ as in Section 3a.)

The preimage of $\bar{X}$ in $G_{v}$ is an almost invariant set $X_{v}$ over the preimage $H_{0}$ of $\bar{H}_{0}$. We extend it to an almost invariant set $X$ of $G$ as follows. Let $S^{\prime}$ be the set of vertices $u \neq v$ of $T$ such that, denoting by $e$ the initial edge of the segment $[v, u]$, the geodesic of $\mathbb{M}^{2}$ invariant under $G_{e} \subset G_{v}$ lies in $P^{+}$; note that it lies in either $P^{+}$or $P^{-}$. Then $X$ is the union of $X_{v}$ with the set of $g \notin G_{v}$ such that $g v \in S^{\prime}$.

Starting from $\bar{H}$, we have thus constructed an almost invariant set $X$, which is well defined up to equivalence and complementation (because of the choices of $x_{0}$ and $\left.P^{ \pm}\right)$. We say that $X$ is a $Q H$-almost invariant subset based on $v$. We let $\mathrm{QH}_{v}(T)$ be the set of equivalence classes of $\mathrm{QH}$-almost invariant subsets obtained from $v$ as above (varying $\bar{H}$ ), and we let $\mathrm{QH}(T)$ be the union of all $\mathrm{QH}_{v}(T)$ when $v$ ranges over all $\mathrm{QH}$-vertices of $T$.

Theorem 3.11. With $\mathscr{E}$ and $T$ as in Theorem 3.3, let $\hat{\mathscr{Q}}=\mathscr{B}(T) \cup \mathrm{QH}(T)$. Then $R N(\hat{\mathscr{X}})$ is isomorphic to a subdivision of $T_{c}$.

Proof. The proof is similar to that of Theorem 3.3.

If $X$ is a $\mathrm{QH}$-almost invariant subset as constructed above, we call $S=S^{\prime} \cup\{v\}$ the $Q H$-forest associated to $X$. We say that it is based on $v$. The coboundary of $S$ is infinite, but all its edges contain $v$. We may therefore view $S$ as a subtree of $T$ (the union of $v$ with certain components of $T \backslash\{v\}$ ). It is a union of cylinders. We let $S^{*}=(T \backslash S) \cup\{v\}$, so that $S \cap S^{*}=\{v\}$.

Note that $S$ cannot contain a peripheral special forest $S_{v, Y}$, with $Y$ a cylinder containing $v$ (this is because the subgroup $\bar{H} \subset \Sigma$ was chosen nonperipheral).

Conversely, given a $\mathrm{QH}$-forest $S$, one can recover $H_{0}$, which is the stabilizer of $S$, and the equivalence class of $X$. In other words, there is a bijection between $\mathrm{QH}_{v}(T)$ 
and the set of QH-forests based on $v$. We denote by $X_{S}$ the almost invariant set $X$ corresponding to $S$ (it is well defined up to equivalence). Note that $X_{S}$ is not a subset of $\{g \in G \mid g v \in S\}$, and these sets have the same intersection with $G \backslash G_{v}$.

The following fact is analogous to Lemma 3.5.

Lemma 3.12. Let $S$ be a $Q H$-forest based on $v$. Let $S^{\prime}$ be a nontrivial special forest, or a $Q H$-forest based on $v^{\prime} \neq v$.

(1) $X_{S} \cap X_{S^{\prime}}$ is small if and only if $S \cap S^{\prime}=\varnothing$.

(2) $X_{S}$ and $X_{S^{\prime}}$ do not cross.

Proof. When $S^{\prime}$ is a special forest, we use $v$ as a basepoint to define $X_{S^{\prime}}$ as the set $\left\{g \mid g v \in S^{\prime}\right\}$. Beware that $X_{S}$ is properly contained in $\{g \mid g v \in S\}$.

We claim that if $S^{\prime}$ is a special forest with $v \notin S^{\prime}$ and $S \cap S^{\prime} \neq \varnothing$, then $X_{S^{\prime}} \subset X_{S}$. Let $Y^{\prime}$ be the cylinder on which $S^{\prime}$ is based. Since each connected component of $S^{\prime}$ contains a point in $Y^{\prime}$, there is a point $w \neq v$ in $S \cap Y^{\prime}$. Since $S$ is a union of cylinders, $S$ contains $Y^{\prime}$. All connected components of $S^{\prime}$ therefore contain a point of $S$ and so are contained in $S \backslash\{v\}$ since $v \notin S^{\prime}$. We deduce $X_{S^{\prime}} \subset X_{S}$.

We now prove (1). If $S \cap S^{\prime}=\varnothing$, then $X_{S} \cap X_{S^{\prime}}=\varnothing$. We assume $S \cap S^{\prime} \neq \varnothing$, and we show that $X_{S} \cap X_{S^{\prime}}$ is not small. If $S^{\prime}$ is a QH-forest, then $v \in S^{\prime}$ or $v^{\prime} \in S$. If for instance $v \in S^{\prime}$, then $X_{S} \cap X_{S^{\prime}}$ is not small because it contains $X_{S} \cap G_{v}$. Now assume that $S^{\prime}$ is a special forest. If $v \in S^{\prime}$, the same argument applies, so assume that $v \notin S^{\prime}$. The claim implies $X_{S^{\prime}} \subset X_{S}$, so $X_{S} \cap X_{S^{\prime}}$ is not small.

To prove (2), first consider the case where $S^{\prime}$ is a QH-forest. Up to changing $S$ and $S^{\prime}$ to $S^{*}$ or $S^{* *}$, one can assume $S \cap S^{\prime}=\varnothing$, so $X_{S}$ does not cross $X_{S^{\prime}}$. If $S^{\prime}$ is a special forest, we can assume $v \notin S^{\prime}$ by changing $S^{\prime}$ to $S^{\prime *}$. By the claim, $X_{S}$ does not cross $X_{S^{\prime}}$.

The lemma implies that no element of $\mathrm{QH}(T)$ crosses an element of $\mathscr{B}(T)$, and elements of $\mathrm{QH}_{v}(T)$ do not cross elements of $\mathrm{QH}_{v^{\prime}}(T)$ for $v \neq v^{\prime}$.

Since $\mathrm{QH}_{v}(T)$ is a cross-connected component, the set $\hat{\mathscr{H}}$ of cross-connected components of $\mathscr{B}(T) \cup \mathrm{QH}(T)$ is therefore the set of cross-connected components of $\mathscr{B}(T)$, together with one new cross-connected component $\mathrm{QH}_{v}(T)$ for each $\mathrm{QH}$ vertex $v$.

One extends the map $\Phi$ defined in the proof of Theorem 3.3 to a map $\hat{\Phi}: \hat{\mathscr{H}} \rightarrow T_{c}$ by sending $\mathrm{QH}_{v}(T)$ to $v$ (viewed as a vertex of $V_{0}\left(T_{c}\right)$ since a $\mathrm{QH}$-vertex belongs to infinitely many cylinders). We need to prove that $\hat{\Phi}$ preserves betweenness.

Lemmas 3.9 and 3.10 extend immediately to the case where $S^{\prime}$ is a $\mathrm{QH}$-forest: one just needs to define $\eta_{Y}\left(S^{\prime}\right)=\operatorname{pr}_{Y}\left(v^{\prime}\right)$ for $S^{\prime}$ based on $v^{\prime}$, so that $v^{\prime}$ plays the role of $Y^{\prime}$ in the proofs. (In the proof of Lemma 3.9, the assertion that $\eta \notin S^{\prime}$ should be replaced by the fact that $S^{\prime} \cap Y$ contains no edge; this holds since otherwise $S^{\prime}$ would contain $Y$.) This allows to prove that, if $C_{2}$ is not a component $\mathrm{QH}_{v}(T)$, then $\Phi\left(C_{2}\right)$ is between $\Phi\left(C_{1}\right)$ and $\Phi\left(C_{3}\right)$ if and only if $C_{2}$ lies between $C_{1}$ and $C_{3}$. 
To treat the case when $C_{2}=\mathrm{QH}_{v}(T)$, we need a cylinder-valued projection $\eta_{v}$. Let $Y$ be a cylinder or a $\mathrm{QH}$-vertex distinct from $v$. We define $\eta_{v}(Y)$ as the cylinder of $T$ containing the initial edge of $[v, x]$ for any $x \in Y$ different from $v$. Equivalently, $\eta_{v}(Y)$ is $Y$ if $v \in Y$, the cylinder containing the initial edge of the bridge joining $x$ to $Y$ otherwise.

If $v$ lies in a cylinder $Y^{0}$, denote by $\eta_{v}^{-1}\left(Y^{0}\right)$ the union of cylinders $Y$ such that $\eta_{v}(Y)=Y^{0}$. Equivalently, this is the set of points $x \in T$ such that $x=v$ or $[x, v]$ contains an edge of $Y^{0}$.

As before, $\llbracket S \rrbracket$ denotes the cross-connected component represented by $X_{S}$.

Lemma 3.13. Let $S$ be a $Q H$-forest based on $v$. Let $S^{\prime}$ be a nontrivial special forest, or a $Q H$-forest based on $v^{\prime} \neq v$. Let $Y^{\prime}$ be the cylinder or $Q H$-vertex on which $S^{\prime}$ is based, and let $Y^{\prime 0}=\eta_{v}\left(Y^{\prime}\right)$.

If $S^{\prime} \subset S$, then $S^{\prime} \subset \eta_{v}^{-1}\left(Y^{\prime 0}\right) \subset S$.

Moreover, $\left.\Phi\left(\left[S^{\prime}\right]\right]\right)$ and $Y_{c}^{\prime 0}$ lie in the same component of $\left.\left.T_{c} \backslash\{\Phi(\llbracket S]]\right)\right\}$.

We leave the proof of this lemma to the reader.

Assume now that $S_{1} \subset S_{2} \subset S_{3}$ with $\llbracket S_{i} \rrbracket=C_{i}$ and $S_{2}$ based on $v$. For $i=1,3$, let $Y_{i}^{0}=\eta_{v}\left(Y_{i}\right)$. Then $S_{1} \subset \eta_{v}^{-1}\left(Y_{1}^{0}\right) \subset S_{2}$ and $S_{3}^{*} \subset \eta_{v}^{-1}\left(Y_{3}^{0}\right) \subset S_{2}^{*}$. In particular, $Y_{1}^{0} \neq Y_{3}^{0}$. Since $\left(Y_{1}^{0}\right)_{c}$ and $\left(Y_{3}^{0}\right)_{c}$ are neighbors of $v_{c}$, they lie in distinct components of $T_{c} \backslash\left\{\Phi\left(C_{2}\right)\right\}$. By Lemma 3.13, so do $\Phi\left(\llbracket S_{1} \rrbracket\right)$ and $\Phi\left(\llbracket S_{3} \rrbracket\right)$.

Conversely, assume that $C_{2}$ does not lie between $C_{1}$ and $C_{3}$, and consider $S_{1} \subset S_{2}$ and $S_{3} \subset S_{2}$ with $\llbracket\left[S_{i} \rrbracket=C_{i}\right.$. For $i=1,3$, let $Y_{i}^{0}$ be as above. If $Y_{1}^{0}=Y_{3}^{0}$, then $\Phi\left(C_{2}\right)$ is not between $\Phi\left(C_{1}\right)$ and $\Phi\left(C_{3}\right)$ by Lemma 3.13, and we are done. If $Y_{1}^{0} \neq Y_{3}^{0}$, these cylinders correspond to distinct peripheral subgroups of $G_{v}$, with invariant geodesics $\gamma_{1} \neq \gamma_{3}$. There exists a nonperipheral group $\bar{H} \subset \Sigma$, as in the beginning of this subsection, whose invariant geodesic separates $\gamma_{1}$ and $\gamma_{3}$. Let $S_{2}^{\prime}$ be the associated QH-forest. Then $\left[\left[S_{2}^{\prime}\right]\right]=C_{2}$ and, up to complementation, $\eta_{v}^{-1}\left(Y_{1}^{0}\right) \subset S_{2}^{\prime}$ and $\eta_{v}^{-1}\left(Y_{3}^{0}\right) \subset S_{2}^{\prime *}$. It follows that $S_{1} \subset S_{2}^{\prime}$ and $S_{3}^{*} \subset S_{2}^{\prime *}$, so $C_{2}$ lies between $C_{1}$ and $C_{3}$, contradicting our assumptions.

\section{The regular neighborhood of Scott and Swarup}

A group is $\mathrm{VPC}_{n}$ if some finite index subgroup is polycyclic of Hirsch length $n$. For instance, $\mathrm{VPC}_{0}$ groups are finite groups, $\mathrm{VPC}_{1}$ groups are virtually cyclic groups, and $\mathrm{VPC}_{2}$ groups are virtually $\mathbb{Z}^{2}$ (but not all $\mathrm{VPC}_{n}$ groups are virtually abelian for $n \geq 3$ ).

Fix $n \geq 1$. We assume that $G$ is finitely presented and does not split over a $\mathrm{VPC}_{n-1}$ subgroup. We also assume that $G$ itself is not $\mathrm{VPC}_{n+1}$. All trees we consider here are assumed to have $\mathrm{VPC}_{n}$ edge stabilizers.

A subgroup $H \subset G$ is universally elliptic if it is elliptic in every tree. A tree is universally elliptic if all its edge stabilizers are. 
A tree is a JSJ tree (over $\mathrm{VPC}_{n}$ subgroups) if it is universally elliptic, and maximal for this property: it dominates every universally elliptic tree. JSJ trees exist (because $G$ is finitely presented) and belong to the same deformation space, called the JSJ deformation space; see [Guirardel and Levitt 2009].

A vertex stabilizer $G_{v}$ of a JSJ tree is flexible if it is not $\mathrm{VPC}_{n}$ and is not universally elliptic. It follows from [Dunwoody and Sageev 1999] that a flexible vertex stabilizer is a QH-subgroup, as defined in Section 3e: There is an exact sequence $1 \rightarrow F \rightarrow G_{v} \rightarrow \Sigma \rightarrow 1$, where $\Sigma=\pi_{1}(\mathcal{O})$ is the fundamental group of a hyperbolic 2-orbifold, $F$ is $\mathrm{VPC}_{n-1}$, and every incident edge group $G_{e}$ is peripheral. Note that the $\mathrm{QH}$-almost invariant subsets $X$ constructed in Section $3 \mathrm{e}$ are over $\mathrm{VPC}_{n}$ subgroups.

We can now describe the regular neighborhood of all almost invariant subsets of $G$ over $\mathrm{VPC}_{n}$ subgroups as a tree of cylinders.

Theorem 4.1. Let $G$ be a finitely presented group, and let $n \geq 1$. Assume that $G$ does not split over a $\mathrm{VPC}_{n-1}$ subgroup and that $G$ is not $\mathrm{VPC}_{n+1}$. Let $T$ be a JSJ tree over $\mathrm{VPC}_{n}$ subgroups, and let $T_{c}$ be its tree of cylinders for the commensurability relation.

Then Scott and Swarup's regular neighborhood of all almost invariant subsets over $\mathrm{VPC}_{n}$ subgroups is equivariantly isomorphic to a subdivision of $T_{c}$.

This is immediate from Theorem 3.11 and Theorem 4.2, which says one can read any almost invariant set over a $\mathrm{VPC}_{n}$ subgroup in a JSJ tree $T$, and which follows from [Dunwoody and Swenson 2000] and [Scott and Swarup 2003, Theorem 8.2].

Theorem 4.2. Let $G$ and $T$ be as above.

For any almost invariant subset $X$ over a $\mathrm{VPC}_{n}$ subgroup, the equivalence class $[X]$ belongs to $\mathscr{B}(T) \cup \mathrm{QH}(T)$.

Proof. We essentially follow the proof by Scott and Swarup [2003], ${ }^{1}$ and we adopt their definitions. All trees considered here have $\mathrm{VPC}_{n}$ edge stabilizers.

Let $X$ be a nontrivial almost invariant subset over a $\mathrm{VPC}_{n}$ subgroup $H$. We first consider the case where $X$ crosses strongly some other almost invariant subset. Then by [Dunwoody and Swenson 2000, Proposition 4.11], $H$ is contained as a nonperipheral subgroup in a QH-vertex stabilizer $W$ of some tree $T^{\prime}$. When acting on $T$, the group $W$ fixes a QH-vertex $v \in T$; see [Guirardel and Levitt 2009, Remark 7.20].

Note that $H$ is not peripheral in $G_{v}$, because it is not peripheral in $W$. Since $(G, H)$ only has 2 coends [2003, Proposition 13.8], there are (up to equivalence) only two almost invariant subsets over subgroups commensurable with $H$ (namely $X$ and $\left.X^{*}\right)$, and therefore $[X] \in \mathrm{QH}_{v}(T)$.

\footnotetext{
${ }^{1}$ From here on, [2003] refers to [Scott and Swarup 2003].
} 
From now on, we assume that $X$ crosses strongly no other almost invariant subset over a $\mathrm{VPC}_{n}$ subgroup. Then, by [Dunwoody and Roller 1993] and [Dunwoody and Swenson 2000, Section 3], there is a nontrivial tree $T_{0}$ with one orbit of edges and an edge stabilizer $H_{0}$ commensurable with $H$.

Since $X$ crosses strongly no other almost invariant set, $H$ and $H_{0}$ are universally elliptic; see [Guirardel 2005, Lemme 11.3]. In particular, $T$ dominates $T_{0}$. It follows that there is an edge of $T$ with stabilizer contained in $H_{0}$ (necessarily with finite index). This edge is contained in a cylinder $Y$ associated to the commensurability class of $H$.

The main case is when $T$ has no edge $e$ such that $Z_{e}$ crosses $X$. (See Section 3a for the definition of $Z_{e}$.) The following lemma implies that $X$ is enclosed in some vertex $v$ of $T$.

Lemma 4.3. Suppose $G$ is finitely generated. Let $X \subset G$ be a nontrivial almost invariant set over a finitely generated subgroup $H$. Let $T$ be a tree with an action of $G$. If $X$ crosses no $Z_{e}$, then $X$ is enclosed in some vertex $v \in T$.

Proof. The argument follows a part of the proof of [2003, Proposition 5.7].

Given two almost invariant subsets, we use the notation $X \geq Y$ when $Y \cap X^{*}$ is small. The noncrossing hypothesis says that each edge $e$ of $T$ may be oriented so that $Z_{e} \geq X$ or $Z_{e} \geq X^{*}$. If one can choose both orientations for some $e$, then $X$ is equivalent to $Z_{e}$, so $X$ is enclosed in both endpoints of $e$ and we are done.

We orient each edge of $T$ in this manner. We color the edge blue or red according to whether $Z_{e} \geq X$ or $Z_{e} \geq X^{*}$. No edge can have both colors. If $e$ is an oriented edge, and if $e^{\prime}$ lies in $T_{e}^{*}$, then $e^{\prime}$ is oriented towards $e$, so that $Z_{e} \subset Z_{e^{\prime}}$, and $e^{\prime}$ has the same color as $e$. In particular, given a vertex $v$, either all edges containing $v$ are oriented towards $v$, or there exists exactly one edge containing $v$ and oriented away from $v$, and all edges containing $v$ have the same color.

If $v$ is as in the first case, $X$ is enclosed in $v$ by definition. If there is no such $v$, then all edges have the same color and are oriented towards an end of $T$. By [2003, Lemma 2.31], $G$ is contained in the $R$-neighborhood of $X$ for some $R>0$, so $X$ is trivial, a contradiction.

Let $v$ be a vertex of $T$ enclosing $X$. In particular, $H \subset G_{v}$. The set $X_{v}=X \cap G_{v}$ is an $H$-almost invariant subset of $G_{v}$ (note that $G_{v}$ is finitely generated). By [2003, Lemma 4.14], there is a subtree $S \subset T$ containing $v$, with $S \backslash\{v\}$ a union of components of $T \backslash\{v\}$, such that $X$ is equivalent to $X_{v} \cup\{g \mid g . v \in S \backslash\{v\}\}$.

Lemma 4.4. The $H$-almost invariant subset $X_{v}$ of $G_{v}$ is trivial.

Proof. Otherwise, by [Dunwoody and Roller 1993; Dunwoody and Swenson 2000], there is a $G_{v}$-tree $T_{1}$ with one orbit of edges and an edge stabilizer $H_{1}$ commensurable with $H$, and an edge $e_{1} \subset T_{1}$, such that $Z_{e_{1}}$ lies up to equivalence in the Boolean algebra generated by the orbit of $X_{v}$ under the commensurator of $H$ in $G_{v}$. 
Note that $G_{e}$ is elliptic in $T_{1}$ for each edge $e$ of $T$ incident to $v$ : By symmetry of strong crossing [2003, Proposition 13.3], $G_{e}$ does not cross strongly any translate of $X$, and thus does not cross strongly $Z_{e_{1}}$, so $G_{e}$ is elliptic in $T_{1}$ [Guirardel 2005, lemme 11.3]. This ellipticity allows us to refine $T$ by creating new edges with stabilizer conjugate to $H_{1}$. Since $H_{1}$ is universally elliptic, this contradicts the maximality of the JSJ tree $T$.

After replacing $X$ by an equivalent almost invariant subset or its complement, and possibly changing $S$ to $(T \backslash S) \cup\{v\}$, we can assume that $X=\{g \mid g . v \notin S\}$. Recall that $Y$ is the cylinder defined by the commensurability class of $H$.

Lemma 4.5. The coboundary $\delta S$, consisting of edges $v w$ with $w \notin S$, is a finite set of edges of $Y$.

This implies that $[X] \in \mathscr{B}(T)$, ending the proof when $X$ crosses no $Z_{e}$.

Proof of Lemma 4.5. Let $E$ be the set of edges of $\delta S$, oriented so that $X=\bigsqcup_{e \in E} Z_{e}$ (we use $v$ as a basepoint to define $Z_{e}$ ). Let $A$ be a finite generating system of $G$ such that, for all $a \in A$, the open segment $(a v, v)$ does not meet the orbit of $v$. One can construct such a generating system from any finite generating system by iteratively replacing $\{a\}$ by the pair $\left\{g, g^{-1} a\right\}$ if $(a v, v)$ contains some $g . v$.

Let $\Gamma$ be the Cayley graph of $(G, A)$. For any subset $Z \subset G$, denote by $\delta Z$ the set of edges of $\Gamma$ having one endpoint in $Z$ and the other endpoint in $G \backslash Z$. By our choice of $A$, no edge joins a vertex of $Z_{e}$ to a vertex of $Z_{e^{\prime}}$ for $e \neq e^{\prime}$. It follows that $\delta X=\bigsqcup_{e \in E} \delta Z_{e}$.

Since $\delta X$ is $H$-finite, the set $\delta Z_{e}$ is $H$-finite for each $e \in E$, and $E$ is contained in a finite union of $H$-orbits. Let $e \in E$. Since $\delta Z_{e}$ is $G_{e}$-invariant and $H$-finite, $G_{e} \cap H$ has finite index in $G_{e}$. Since $G_{e}$ and $H$ are both $\mathrm{VPC}_{n}$, they are commensurable, so the $H$-orbit of $e$ is finite. It follows that $E \subset Y$ and that $E$ is finite.

We now turn to the case when $X$ crosses some of the $Z_{e}$. For each $e \in E(T)$, the intersection number $i\left(Z_{e}, X\right)$ is finite [Scott 1998], which means that there are only finitely many edges $e^{\prime}$ in the orbit of $e$ such that $Z_{e^{\prime}}$ crosses $X$. Since $T / G$ is finite, let $e_{1}, e_{1}^{-1}, e_{2}, e_{2}^{-1}, \ldots, e_{n}, e_{n}^{-1}$ be the finite set of oriented edges $e$ such that $Z_{e}$ crosses $X$, where we denote by $e \mapsto e^{-1}$ the orientation-reversing involution. Note that $e_{i} \subset Y$ by [2003, Proposition 13.5]. Now $X$ is a finite union of sets of the form $X^{\prime}=X \cap Z_{e_{1}^{ \pm 1}} \cap \cdots \cap Z_{e_{n}^{ \pm 1}}$. Since $X^{\prime}$ does not cross any $Z_{e}$, its equivalence class lies in $\mathscr{B}(T)$ by the argument above and so does $[X]$.

\section{References}

[Dunwoody and Roller 1993] M. J. Dunwoody and M. A. Roller, "Splitting groups over polycyclicby-finite subgroups”, Bull. London Math. Soc. 25:1 (1993), 29-36. MR 93h:20029 Zbl 0737.20012 
[Dunwoody and Sageev 1999] M. J. Dunwoody and M. E. Sageev, "JSJ-splittings for finitely presented groups over slender groups”, Inventiones Math. 135:1 (1999), 25-44. MR 2000b:20050 Zbl 0939.20047

[Dunwoody and Swenson 2000] M. J. Dunwoody and E. L. Swenson, "The algebraic torus theorem”, Invent. Math. 140:3 (2000), 605-637. MR 2001d:20039 Zbl 1017.20034

[Forester 2002] M. Forester, "Deformation and rigidity of simplicial group actions on trees", Geom. Topol. 6 (2002), 219-267. MR 2003m:20030 Zbl 1118.20028

[Forester 2003] M. Forester, "On uniqueness of JSJ decompositions of finitely generated groups", Comment. Math. Helv. 78:4 (2003), 740-751. MR 2005b:20075 Zbl 1040.20032

[Guirardel 2004] V. Guirardel, "Limit groups and groups acting freely on $\mathbb{R}^{n}$-trees", Geom. Topol. 8 (2004), 1427-1470. MR 2005m:20060 Zbl 1114.20013

[Guirardel 2005] V. Guirardel, "Cœur et nombre d'intersection pour les actions de groupes sur les arbres”, Ann. Sci. École Norm. Sup. (4) 38:6 (2005), 847-888. MR 2007e:20055 Zbl 1110.20019

[Guirardel and Levitt 2007] V. Guirardel and G. Levitt, "Deformation spaces of trees", Groups Geom. Dyn. 1:2 (2007), 135-181. MR 2009a:20041 Zbl 1134.20026

[Guirardel and Levitt 2008] V. Guirardel and G. Levitt, "Trees of cylinders and canonical splittings", preprint, version 1, 2008. arXiv 0811.2383v1

[Guirardel and Levitt 2009] V. Guirardel and G. Levitt, "JSJ decompositions: definitions, existence, uniqueness, I: The JSJ deformation space", preprint, 2009. arXiv 0911.3173

[Scott 1998] P. Scott, “The symmetry of intersection numbers in group theory”, Geom. Topol. 2 (1998), 11-29. MR 99k:20076a Zbl 0897.20029

[Scott and Swarup 2003] P. Scott and G. A. Swarup, Regular neighbourhoods and canonical decompositions for groups, Astérisque 289, Société Mathématique de France, Paris, 2003. Corrections available at http://www.math.lsa.umich.edu/ pscott/regularn/errataregularn.pdf. MR 2005f:20045 Zbl 1036.20028

Received December 9, 2008.

\section{VINCENT GUIRARDEL}

InSTITUT DE MATHÉMATIQUes DE TOUlouse

UNIVERSITÉ DE TOULOUSE ET CNRS (UMR 5219)

118 ROUTE DE NARBONNE

F-31062 TOULOUSE CEDEX 9

FRANCE

guirardel@math.univ-toulouse.fr

www.math.univ-toulouse.fr/ guirardel

\section{GILBERT LEVITT}

LABoratoire de Mathématiques Nicolas Oresme

UNIVERSITÉ DE CAEN ET CNRS (UMR 6139)

BP 5186

F-14032 CAEN CEDEX

FRANCE

levitt@math.unicaen.fr 